\title{
Duck enteritis virus (DEV) UL54 protein, a novel partner, interacts with DEV UL24 protein
}

\author{
Xinghong Gao ${ }^{1,2,3}$, Renyong Jia1,3,4*, Mingshu Wang ${ }^{1,3,4}$, Qiao Yang ${ }^{1,3,4}$, Shun Chen ${ }^{1,3,4}$, Mafeng Liu ${ }^{1,3,4}$, \\ Zhongqiong Yin $^{4}$ and Anchun Cheng ${ }^{1,3,4^{*}}$
}

\begin{abstract}
Background: UL24 is a multifunctional protein that is conserved among alphaherpesviruses and is believed to play an important role in viral infection and replication.

Results: In this paper, to investigate putative UL24-binding proteins and to explore the functional mechanisms of DEV UL24, yeast two-hybrid $(\mathrm{Y} 2 \mathrm{H})$ was carried out, and further verified the interaction between UL24 and partners by co-immunoprecipitation and fluorescence microscopy experiments. Interaction partners of UL24 protein were screened by yeast two-hybrid $(\mathrm{Y} 2 \mathrm{H})$ with the CDNA library of DEV-CHv strain post-infection DEF cells. A novel partner, DEV UL54 protein, was discovered by $\mathrm{Y} 2 \mathrm{H}$ screening and bioinformatic. Co-immunoprecipitation experiments suggested that DEV UL24 interacted with UL54 proteins. And distribution of a part of UL54 protein was changed from nucleus to cytoplasm in DF-1 cells of co-subcellular localization experiments which also showed that DEV UL24 interacted with UL54 proteins.
\end{abstract}

Conclusions: The interaction between the DEV UL24 and UL54 proteins was discovered for the first time. Thus, DEV UL54 protein as a novel partner interacted with DEV UL24 protein.

Keywords: DEV, UL24 protein, UL54 protein, Protein-protein interaction (PPI)

\section{Finding}

Duck enteritis virus (DEV, anatid alphaherpesvirus 1 species), clustered in mardivirus genus, alphaherpesvirinae subfamily, Herpesviridae family according to the latest report of the International Committee on Taxonomy of Viruses (ICTV) [1], causes considerable economic losses to the commercial duck industry and poses a continuous threat to wild and migratory waterfowl populations (e.g., ducks, geese and swans) due to their high mortality and decreased egg production rates [2].

Currently, there are three complete genomic sequences of DEV strains available in GenBank: the Chinese virulent DEV strain (DEV CHv) [3, 4], the European virulent strain (2085) [5], and the attenuated vaccine strain (VAC) [6], and the publications related to the

\footnotetext{
*Correspondence: jiary@sicau.edu.cn; chenganchun@vip.163.com ${ }^{1}$ Avian Disease Research Center, College of Veterinary Medicine, Sichuan Agricultural University, Chengdu, Sichuan 611130, China

Full list of author information is available at the end of the article
}

three genome sequences have cast light on the genome structure of DEV. DEV is a linear, double-stranded DNA virus, the genome size of which is approximately 158$162 \mathrm{~kb}$ [3-6]. The entire genome of DEV is composed of a unique long (UL), a unique short (US) and two inverted repeated sequences (IRS and TRS) [6]. A total of 78 ORFs were predicted to code for the potential functional proteins. Of these ORFs, 10 and 68 ORFs coded for structural proteins and non-structural proteins, respectively. Many DEV proteins, such as UL16 [7], UL38 [8], gE [9], gN [10, 11] have been researched in molecular biology studies. However, these researches on protein-protein interaction (PPI) were only done between $\mathrm{gM}$ and $\mathrm{gN}$ [10]. And there was no report on partners of DEV UL24 protein.

UL24 protein is a conserved multifunctional protein and is believed to play an important role in viral infection and replication. UL24 protein contains five homology domains (HDs) with a high percentage of amino 
acid identity among its homologs of the other Herpesvirus family members (including HSV1/2 UL24, EHV-1 ORF37, HCMV UL76, MHV-68 ORF20, and so on) and one PD-(D/E)XK endonuclease motif in the $\mathrm{N}$-terminal regions (NTRs) [12-14]. Using mouse infection model, researches showed that HSV-1 UL24 protein was involved in viral pathogenesis $[15,16]$ and contributed to viral replication in the mucous membranes [17]. ORF 37 is a neuropathogenic determinant of equine herpesvirus 1 (EHV-1) $[18,19]$. UL76 protein of human cytomegalovirus (HCMV) was able to induce DNA double-strands breaks and DNA damage response [20-22]. ORF 20 of murine herpesvirus 68 (MHV-68) was reported to be involved in inducing cell-cycle arrest at the G2/M phase followed by apoptosis [13, 23]. In summary, UL24 protein contributes to virus virulence $[16,17,19]$, viral replication [15, 24-26], cell membrane fusion [27, 28], cell cycle arrest [23], and redistribution of nucleolin (C23) and nucleophosmin (B23) [27-31]. Up to now, research on DEV UL24 protein showed that it is located in the cytoplasm around the periphery of the nucleus in DEVinfected DEF cells [32]. And attenuated Salmonella Typhimurium delivering DNA vaccine encoding DEV UL24 induced immune responses and conferred good protection against challenge [33, 34].

The DEV UL54 is an immediate early gene [33], but its function is not very clear. Bioinformation analysis showed that DEV UL54 encode a $51.75 \mathrm{KDa}$ protein of 458 AA with $56 \%$ homology to the corresponding HSV1 protein ICP27. ICP27, a conserved and multifunctional protein, is characterized nucleocytoplasmic shuttling based on crucial nuclear localization signal (NLS) and nuclear export signal (NES) [35-37]. ICP27 has been implicated in viral replication $[35,38]$, gene expression [39, 40], apoptosis [41] and host immunization reactions [42, 43], all of which promote infection. Thus, these features of ICP27 provide ideas or inspiration for research on UL54.

UL24 protein family is a multifunctional protein playing important roles in herpesvirus invasion and replication. However, there are only a few reports on the molecular mechanisms underlying the function of UL24 protein $[32,34,44,45]$. Thus, study on PPI of DEV UL24 contributes to better understanding of functions and molecular mechanisms of this protein, which also prompts us to understand the molecular mechanisms of DEV infection. To this end, we employed yeast twohybrid technology coupled with co-immunoprecipitation to screen DEV UL24 protein interacting partner.

Sequence analysis of the $\mathrm{N}$-terminal region of DEV UL24 gene (nucleotides 1-720, Additional file 1: Figure S1) was carried out by codon optimization with host yeast of Saccharomyces cerevisiae (http://www.jcat.de/). Optimized sequence was generated by company of
Huada (China). To clone full-length optimized DEV UL24 gene, two pairs of primer were designed (Table 1, primers UL24/N-F/R, UL24/C-F/R). Viral sequences (Nterminal fragment, UL24/N; and full-length optimized DEV UL24 gene, UL24/FL) were cloned into pGBKT7 plasmid (bait plasmid; Clontech) and Y2HGold strain (bait strains; Clontech) was transformed with this two recombinant plasmids (Fig. 1a, b), respectively. UL24/N strain was used as a control. Then, bait strains were verified for self-activation, toxicity and Western blot analysis according to protocols as described in Matchmaker Gold Yeast Two-Hybrid System User Manual (Clontech) [46, 47].Self-activation and toxicity detection of bait strains were negative. Western blot analysis revealed that UL24/ $\mathrm{N}$ and UL24/FL-fusion proteins were expressed. Based on theoretical estimates, UL24/N and UL24/FL-fusion proteins (contain GAL4 DNA binding domain of pGBKT7 plasmid about $22 \mathrm{kDa}$; myc flag protein about $1 \mathrm{kDa}$ ) are about $50 \mathrm{kDa}, 69 \mathrm{kDa}$ respectively (Fig. 1c).

To explore the functional mechanisms of DEV UL24 and to investigate putative UL24-binding proteins, $\mathrm{Y} 2 \mathrm{H}$ screens were performed by mating (according to protocol in Matchmaker Gold Yeast Two-Hybrid System User Manual, Clontech). Briefly, the cDNA library was constructed by previously described, which was comprised all genes of the DEF cells post-infection DEV-CHv strain and contained more than $10^{7}$ primary clones per milliliter [48]. Four putative interacting proteins, DEV UL54 (Accession: EU071033.1), duck PSF2 (Accession: XM_013096619.1), GNB2L1 (Accession: XM_005018317.2), and Anas platyrhynchos Nudix-type motif 9 (Accession: XM_005012818.2) were obtained by sequencing analysis and NCBI (National Center for Biotechnology Information) blast analysis. NCBI blast analysis suggested that the first base of positive clone contained GNB2L1 mRNA sequence, and matched with the 426th base of GNB2L1 mRNA sequence; the 78th base of positive clone contained Anas platyrhynchos Nudix-type motif 9 mRNA sequence and matched with the 194th base of Anas platyrhynchos Nudix-type motif 9 mRNA sequence. According to triplet code characteristic of nucleic acid code protein, we concluded the positive clones which contained GNB2L1 mRNA sequence and Anas platyrhynchos Nudix-type motif 9 sequences as probably frameshift mutants. PSF2 sequence lay in 3'UTR of duck PSF2 mRNA whereas DEV UL54 sequence unaffected. Thus, we used positive clone which contains UL54 sequence to eliminate the false positive of it by Y2H (Fig. 2). DEV UL54 mRNA also contained a polyA site that was $26 \mathrm{nt}$ downstream of the UL54 CDS region.

DEV UL24 and UL54 sequences were cloned by recombination into $\mathrm{pCMV}$-myc-N and pCMV-Flag-N vectors respectively. Primers were designed and are listed in Table 1 (primers pCMV-myc-UL24 F/R, pCMV-FlagUL54 F/R). Positive clones were identified by 
Table 1 primer sequences

\begin{tabular}{lll}
\hline primer name & sequence & restriction enzyme \\
\hline UL24/N-F & 5'-AGGAGGACCTGCATATGATGGCTTCTAAGGTTCAAAAGAAGAGA-3' & Nde I \\
UL24/N-R & 5'-GGATCCCCGGGAATTCTGGTATTCAGACAAACCAG-3' & EcoR I \\
UL24/C-F & 5'-ATCGCTGGTTGTCTGAATACCACATACCTACCAAAGGTAAGCGCCGG-3' & - \\
UL24/C-R & 5'-GGATCCCCGGGAATTCCTAGTGTTAGTTGGTCTGA & EcoR I \\
pCMV-myc-UL24 F & 5'-ATGGAGGCCCGAATTCGGATGGCATCGAAGGTACAGA-3' & EcoR I \\
pCMV-myc-UL24 R & 5'-GCCGCGGTACCTCGAGACTAGTGTTTAGTTGGTCTGAA-3' & Xho I \\
pCMV-Flag-UL54 F & 5'-CATATGATGGCCTGCAGTGCTAAA-3' & Nde I \\
pCMV-Flag-UL54 R & 5'-GGATCCCAAACATTCATTACAATAAAA-3' & BamH I \\
pEGFP-N1-UL24-F1 & 5'-AAGCTTCGAATTCTGATGGCATCGAAGGTACAGA-3' & EcoR I \\
pEGFP-N1-UL24-R2 & 5'-CGACCGGTGGATCCCGGGCGTGTTTAGTTGGTCTGAATA-3' & Sma I \\
pDsRed-N1-UL54-F1 & 5'-TCTCAAGCTTAAGCTATGGCCTGCAGTGCTAAAC-3' & Hind III \\
pDsRed-N1-UL54R2 & 5'-GGCGACCGGTGAGCTCGTAAACATTTCATTACAATA-3' & BamH I \\
\hline
\end{tabular}

The restriction enzyme sites were bold

sequencing. HEK293T cells (Human embryonic kidney cells, HEK) were cultured in DMEM (Dulbecco modified Eagle medium, Gibco) supplemented with 10\% (v/v) FBS (fetal bovine serum, Gibco), 100 units/mL penicillin, and $100 \mu \mathrm{g} / \mathrm{mL}$ streptomycin in an atmosphere of $5 \% \mathrm{CO}_{2}$ at
$37{ }^{\circ} \mathrm{C}$. For co-expression DEV UL24 and UL54 proteins, equal plasmids were transiently co-transfected into HEK293T cells using a Lipofectamine-2000 transfection reagent system (Invitrogen). Total protein was harvested at $48 \mathrm{~h}$ post-transfection by incubating cells for $30 \mathrm{~min}$
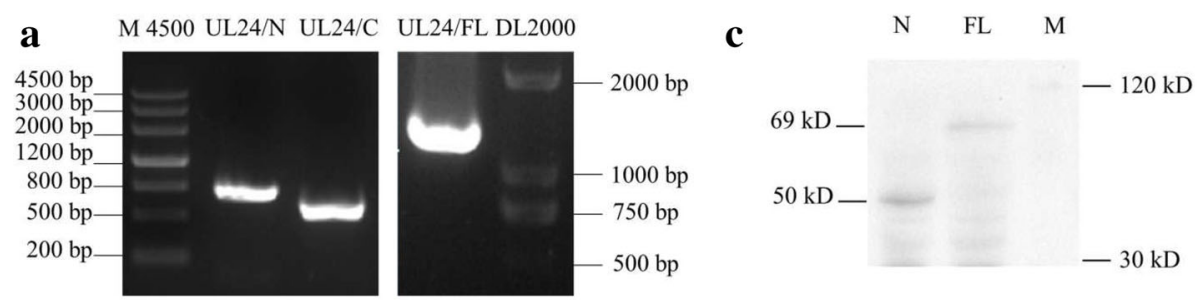

b

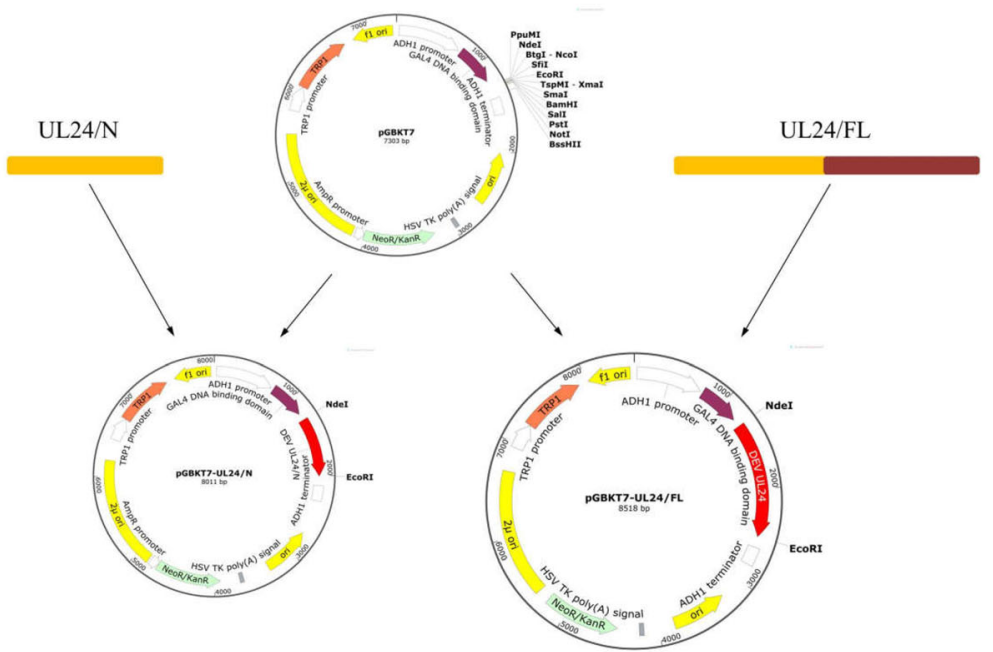

Fig. 1 Bait plasmid construction. a, the nucleotide sequence of UL24/C (721 1230 bp, lane 3) and codon optimized UL24/N (1 720 bp, lane 2) was firstly amplified by PCR. Then, the full length codon optimized UL24 gene (1 $1230 \mathrm{bp}$, lane 4) was amplified with the DNA fragments UL24/C and UL24/N as template by overlap PCR. M4500: DNA Marker $200 \sim 4500$ bp. DL2000: DNA Marker $100 \sim 2000$ bp. b, "bait" proteins (N and FL) were expressed in Saccharomyces cerevisiae Y2HGold strain according to be detected by western blotting. Primary antibody (mouse-anti myc mAb) is diluted 1000 folds, and HRP-goat anti-mouse antibody is diluted 80,000 folds. Immunoreactive proteins are detected using the ECL kit (enhanced chemiluminescence system, Bio-Rad) 


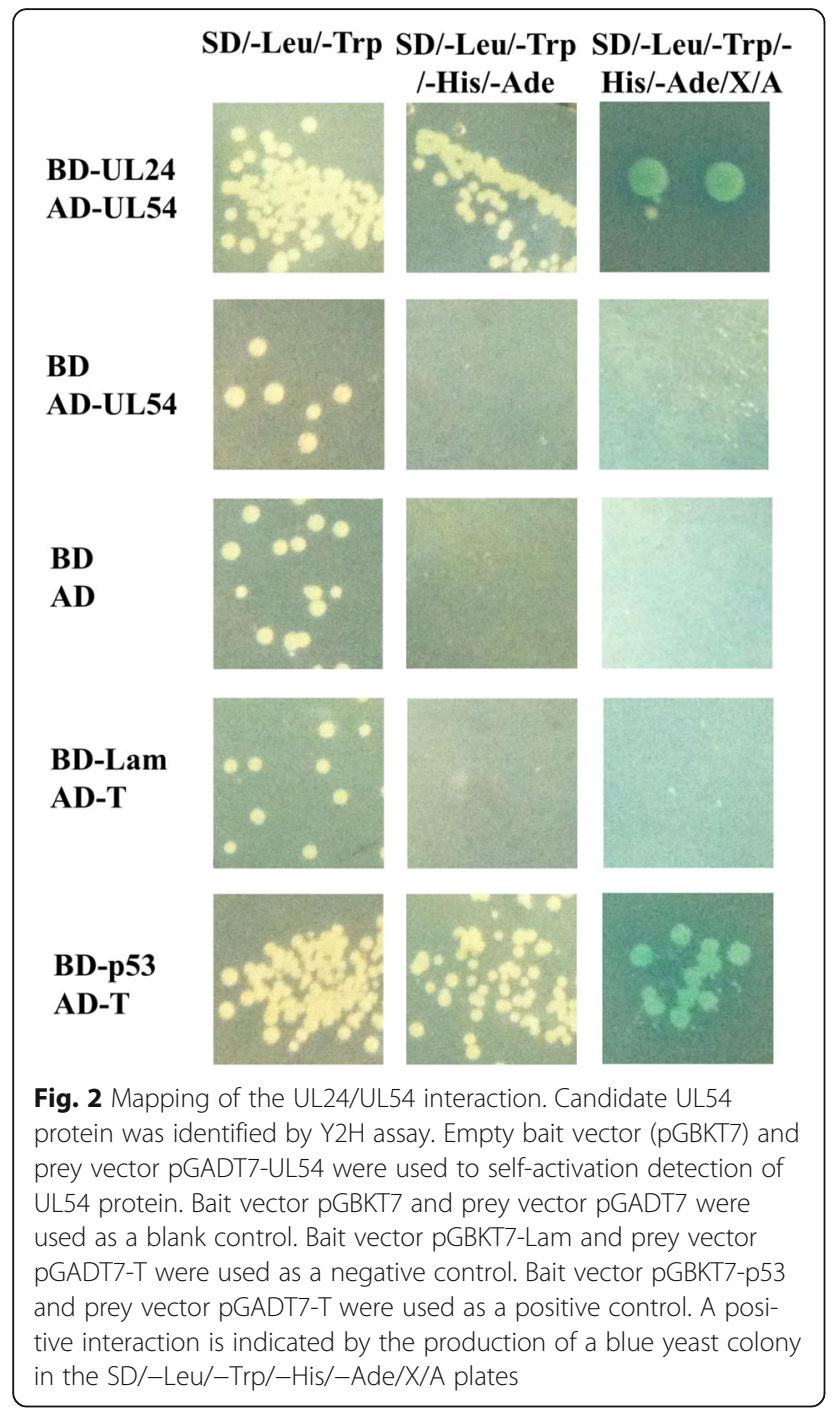

on ice, followed by scraping into NP-40 lysis buffer with the addition of ionic detergents $(0.5 \%$ sodium deoxycholate and $0.1 \%$ SDS) [35]. Debris was pelleted by centrifugation at $13,000 \times \mathrm{g}$ for $20 \mathrm{~min}$ in $4{ }^{\circ} \mathrm{C}$. Western blot (WB) analysis of cell extracts revealed that both UL24 and UL54 fusion proteins were expressed with molecular weights of about $45 \mathrm{kDa}$ and $50.5 \mathrm{kDa}$, respectively (Additional file 2: Figure S2). The full length UL24-fusion protein was expressed and about $69 \mathrm{kDa}$ (containing $22 \mathrm{kDa}$ binding domain of GAL4 protein and $1 \mathrm{kDa}$ myc tagged protein) in Saccharomyces cerevisiae Y2HGold strain firstly according to codon optimization (Additional file 1: Figure S1). Thus, full length DEV UL24 protein was about $46 \mathrm{kDa}$ in Y2HGold strain which was consistent with expected results. Therefore, DEV UL24 protein expressed in eukaryote is about $45 \mathrm{kDa}$.

Immunoprecipitation was performed with $2 \mathrm{mg}$ of total protein incubated with $5 \mu \mathrm{L}$ myc-agarose (mouseanti-myc monoclonal antibody coupling with agarose,
Santa Cruz Biotechnology) for $2 \mathrm{~h}$ at $4{ }^{\circ} \mathrm{C}$, or incubated with $3 \mu \mathrm{g}$ rabbit anti-UL24 antibody (polyclonal antibody, pAb) for $2 \mathrm{~h}$ at $4{ }^{\circ} \mathrm{C}$. And compounds which contained pAb UL24 were incubated with protein A\&G plus agarose (Santa Cruz Biotechnology) for another $2 \mathrm{~h}$ at $4{ }^{\circ} \mathrm{C}$. Then, the other steps of immunoprecipitation were performed as protocol [49]. According to WB analysis, we observed that UL54 fusion protein, in above transfected 293 T cell extracts, was expressed in the experimental group (Fig. 3, lane 1\&2) in contrast to control where no visible band was detected (Fig. 3, lane 3). Simultaneously, cell extracts were precipitated using rabbitanti DEV UL24 antibody coupled to protein A \&Gagarose and precipitated with myc-agarose respectively. Subsequent WB analysis showed that UL54 fusion protein was detected in the experimental group (Fig. 3, lane $1 \& 2$ ), but not in the control group (Fig. 3, lane 3). Furthermore, the intensity of the UL54 fusion protein in lane 2 was greater than in lane 1 . To summarize, using co-immunoprecipitation, our results suggested that UL24 and U54 proteins interact with each other.

To further verify the interaction between UL24 and UL54 protein and to explore the function of UL24 protein during DEV infection, fluorescence microscopy was

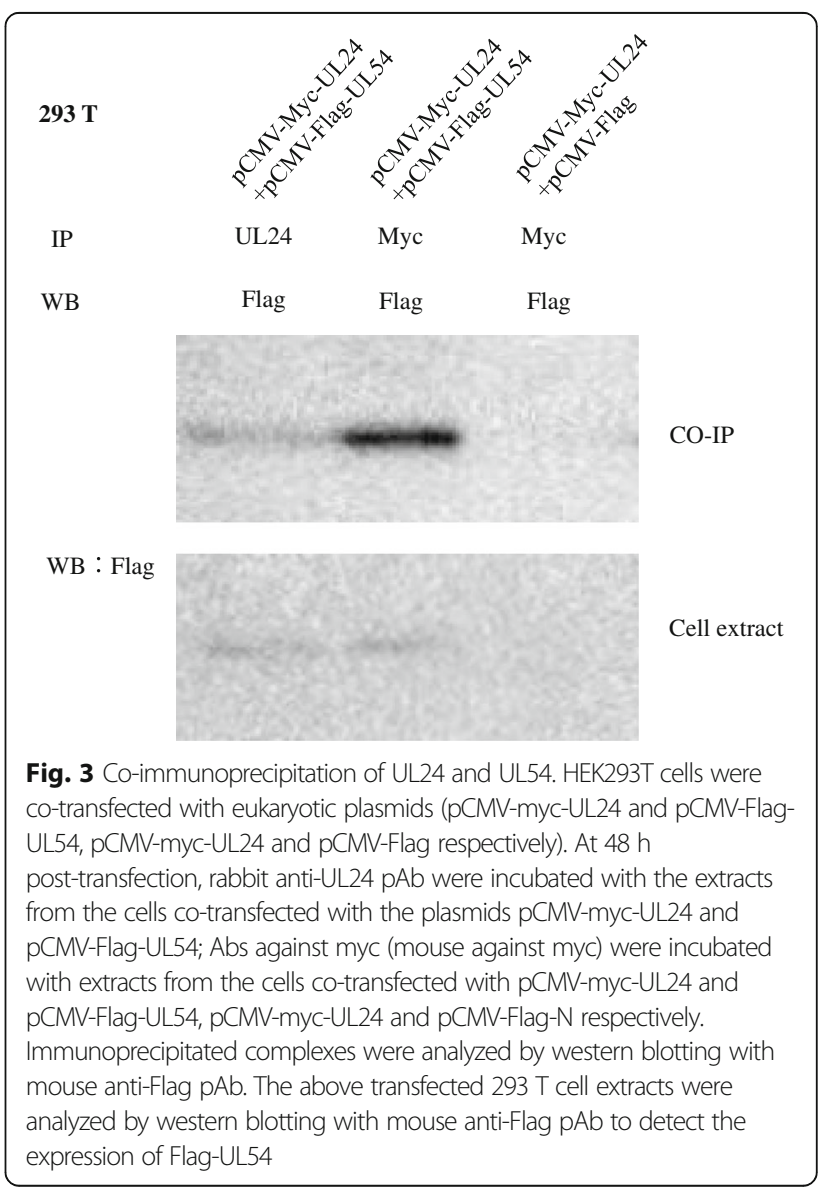


carried out. DEV UL24 and UL54 sequences were cloned by recombination into pEGFP-N1 and pDsRedN1 vectors respectively. Primers were designed as in Table 1 (primers pEGFP-N1-UL24 F/R, pDsRed-N1UL54 F/R). Positive clones were identified by sequencing. Chicken fibroblast cells (DF-1) were cultured and transiently transfected/ co-transfected as same as HEK293T cells. Respectively, 12 h, 24 h, 36 h, 48 h after transfection, the transfected cells were fixed in $4 \%$ paraformaldehyde for $10 \mathrm{~min}$, permeabilized treatment in $0.3 \%$ Triton X-100 for $10 \mu \mathrm{min}$, stained in $10 \mu \mathrm{g} / \mathrm{mL}$ DAPI solution (Sigma) for $8 \mathrm{~min}$, and observed with a fluorescence microscope under a $\times 40$ objective [50]. Results in Fig. 4 also demonstrated that UL24-EGFP protein was localized predominantly to the nucleus but a fraction also appeared to be located in the cytoplasm at $36 \mathrm{~h}$ and $48 \mathrm{~h}$, post-transfection. Simultaneously, significant nuclear fragmentation was observed at $12 \mathrm{~h} 48 \mathrm{~h}$ post-transfection as revealed by DAPI stain. Thirdly, UL24-EGFP protein distribution exhibited a globular shape or crystal shape aggregation. Figure 5 showed that UL54-DsRed protein was located in nucleus predominantly.

Subcellular co-localization of UL24- and UL54-fusion proteins, at $12 \mathrm{~h} \sim 36 \mathrm{~h}$ post-transfection, UL24-EGFP and UL54-DsRed proteins was redistributed equably in nucleus. At $48 \mathrm{~h}$ post-infection, UL54-DsRed proteins were partly transported to cytoplasm although most of UL24- and UL54-fusion proteins were distributed equably in nucleus (Fig. 6, UL24-EGFP + UL54-DsRed group). Furthermore, some cell nucleus, in which UL24EGFP and UL54-DsRed proteins were redistributed equably, was not a form of visible micronucleus by fluorescence microscopy. For in groups of negative control
(UL24-EGFP+ DsRed group, EGFP + UL54-DsRed group), UL24-EGFP and UL54-DsRed proteins were predominantly laid in nucleus, respectively (Fig. 6, list 1\&3). The blank control group (EGFP + DsRed group) revealed that EGFP and DsRed co-located in cytoplasm and nucleus equably (Fig. 6, list 4). In summary, our data suggested that UL24and UL54-fusion protein could be co-expressed in DF-1 cells, and that the redistribution of UL54-fusion protein was caused by interactions between UL24 and UL54 protein.

In the past years, it has been reported that the HSV-1 ICP27 protein, a homologue of DEV UL54 protein [38, 51-53], can shuttle from the nucleus to the cytoplasm due to interaction with the host Nup62 protein [35]. The interactions between ICP27 protein and Nup62 protein inhibits host mRNAs exported to cytoplasm and regulates the expression of virus genes via regulation of the amount of the virus mRNAs exported to cytoplasm $[35,54]$. HSV-1 UL24 protein is located in the nucleus, nucleolus and cytoplasm [55], whereas DEV UL24 protein is localized in the cytoplasm and the nucleus [34, 44]. UL24 protein interaction with UL54 protein existed in other five herpesviruses [56]. Therefore, we infer that the function of UL24 and UL54 PPI in DEV was probably the same as UL24 and ICP27 in HSV-1.

The UL24 protein is located differently in different cells. In this article, the localization of UL24-EGFP fusion protein was in the nucleus and cytoplasm in DF-1 cells (Fig. 4). In previous study, DEV UL24-LTB fusion protein was also located in the nucleus and cytoplasm when it was transiently expressed in COS-7 cells [34]. When overexpressed in COS-7 or DF-1 cells, UL24fusion protein was predominantly localized in the nucleus. But DEV UL24 protein was located in the

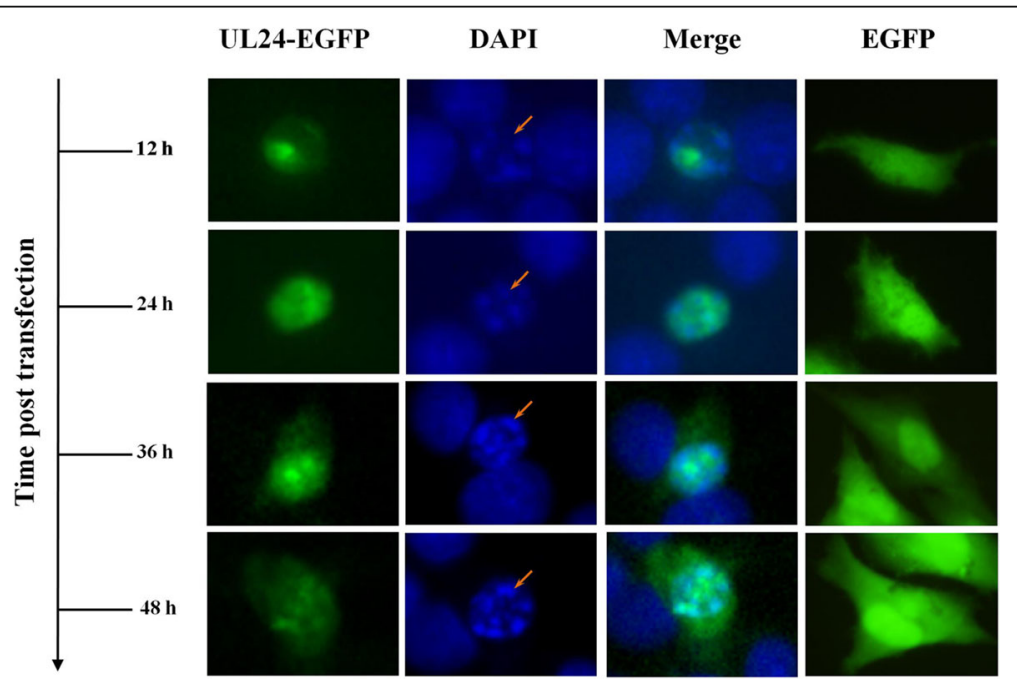

Fig. 4 Subcellular localization of UL24 protein in DF-1 cells. DF-1 cells were transfected with pEGFP-N1-UL24. $12 \mathrm{~h}, 24 \mathrm{~h}, 36 \mathrm{~h}$ and $48 \mathrm{~h}$ after transfection, cells were fixed, permeabilized and then stained with 4, 6-diamidino-2-phenylindole (DAPI). The subcellular localization of UL24 protein was visualized using fluorescent microscopy. The DF-1 cells were transfected with the empty vector pEGFP-N1 as a negative control 


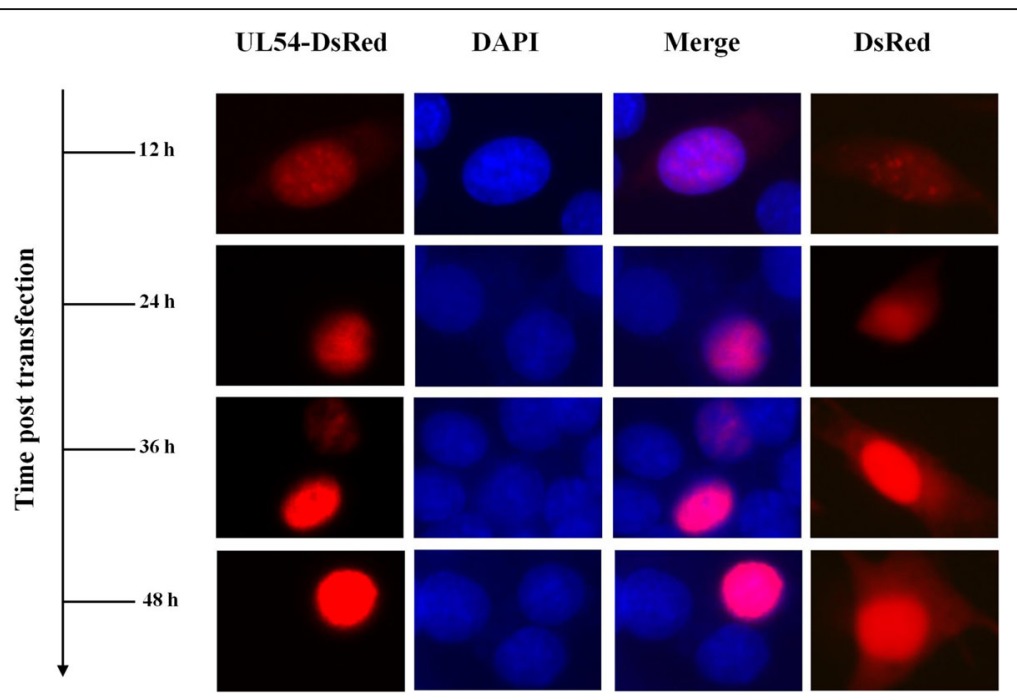

Fig. 5 Subcellular localization of UL54 protein in DF-1 cells. After infection pDsRed-N1-UL54 plasmids $12 \mathrm{~h}, 24 \mathrm{~h}, 36 \mathrm{~h}$ and $48 \mathrm{~h}$ respectively, DF-1 were fixed, hyalinized and DAPI stained. And fluorescence microscopy was visualized directly. Meanwhile, DF-1 cells were transfected with pDsRed-N1 as a negative control

perinuclear region in DEV infected DEF cells, and regardless of an earlier or later time-point in infection, a little UL24 protein was observed in the nucleus [32]. It was guessed that there was a protein which interacted with DEV UL24, and UL24 could made it shuttle from nucleus to cytoplasm during DEV infection. DEV UL54 protein had a characterization of nucleocytoplasmic shuttling [57], and DEV UL24 interacted with UL54. Thus, we concluded that DEV UL54 probably promoted UL24 transportation from nucleus to cytoplasm during DEV infection.
Overexpression of UL24-fusion protein in DF-1 cell, induced DNA fragmentation and formation micronucleus according to DAPI stain (Fig. 5 and Additional file 2: Figure S2), suggested DEV UL24 protein functions in DNA damage. Similarly, HCMV UL76 protein, a homologue of DEV UL24 protein in herpesvirus family could induce DNA fragmentation and a form of micronucleus [21, 22]. Cosubcellular localization of UL24 and UL54-fusion protein, and redistribution of UL24-EGFP and UL54-DsRed proteins were changed at time post-transfection. Thus, we concluded that the redistribution of UL24 and UL54-fusion protein

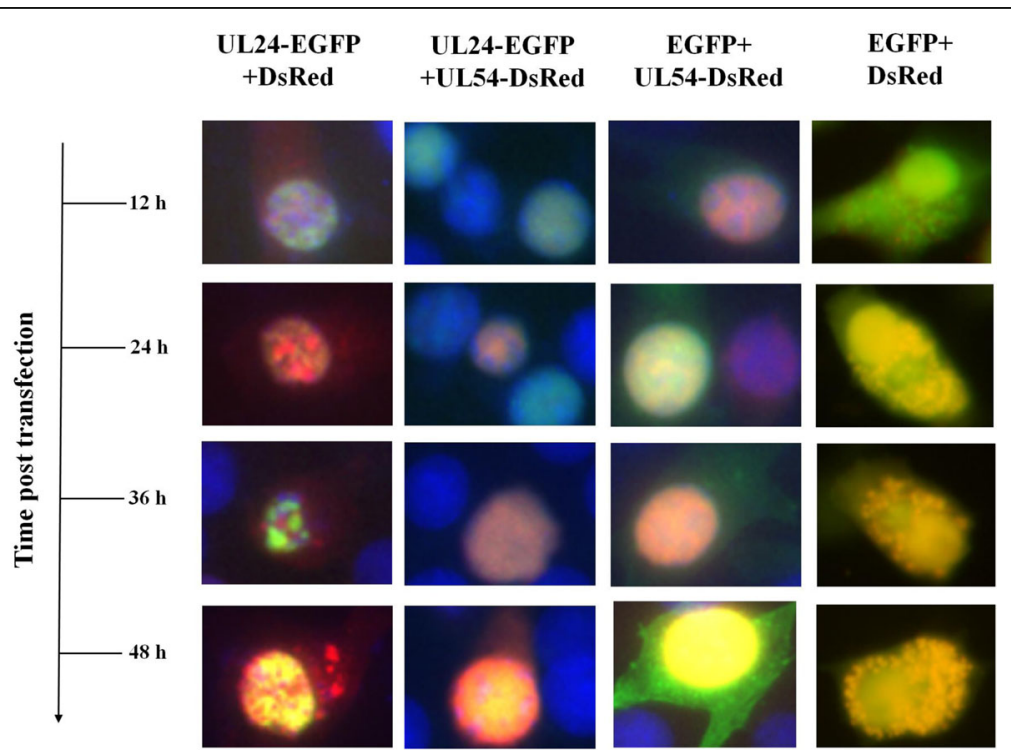

Fig. 6 Subcellular co-localization of UL24 and UL54 protein in DF-1 cells. DF-1 cells were co-transfected with equal pEGFP-N1-UL24 and pDsRedN1, pEGFP-N1 and pDsRed-N1-UL54, pEGFP-N1-UL24 and pDsRed-N-UL54, pEGFP-N1 and pDsRed-N1 respectively. 12 h, 24 h, 36 h and 48 h after infection, cells were fixed, hyalinized and DAPI stained. Fluorescence microscopy was visualized directly 
was caused by interactions between UL24 and UL54 protein. Interestingly, in cells of co-expression UL24 and UL54 proteins, some nucleus did not have DNA damage (Fig. 6, list 2). It suggested that the PPI between UL24 and UL54 protein could reduce the effect of DNA damage, and we would explore the molecular mechanism in the future.

\section{Conclusions}

UL24 is a multifunctional protein, playing important roles in virus invasion and replication. To identify the molecular mechanisms underlying the function of UL24 protein, $\mathrm{Y} 2 \mathrm{H}$ experiment coupled with CO-IP and co-subcellular localization were employed. UL54 protein, as a novel partner, interacted with DEV UL24 protein and conserved in herpesviridae family. In addition, the redistribution of partial UL54 proteins took changes from nucleus to cytoplasm, and the micronucleus disappeared in some of co-expression DF1 cells. We concluded that the interaction between the two proteins is associated with several pathogenic processes in DEV infection, such as DNA damage and viral replication. And the molecular mechanism of this interaction contribution to DEV pathogenic infection is required to be further researched in the future.

\section{Additional files}

Additional file 1: Figure S1. UL24/N gene codon optimization (1 720 bp). The green short-line was optimized nucleotides. (PDF $312 \mathrm{~kb}$ )

Additional file 2: Figure S2. WB analyzed the expression of UL24fusion protein and UL54-fusion protein in HEK293T cells. HEK293T cells were transfected with eukaryotic plasmid pCMV-myc, pCMV-myc-UL24, and pCMV-Flag-UL54 respectively. At $48 \mathrm{~h}$ post-infection, the $293 \mathrm{~T}$ cell extracts were carried out Western blotting analysis, which indicated that myc-UL24 and Flag-UL54 was expressed in 293 T cells and the molecular mass of fusion protein is about $45 \mathrm{KD}, 50.5 \mathrm{KD}$ respectively. Primary Abs against myc-UL24 and Flag-UL54 were serums of rabbit against UL24 and mouse against Flag respectively. (PDF $44 \mathrm{~kb}$ )

\section{Abbreviations \\ CO-IP: Co-immunoprecipitation; CPE: Cytopathic effect; DEV: Duck enteritis virus; DMEM: Dulbecco modified Eagle medium; EHV-1: Equine herpesvirus 1; FBS: Fetal bovine serum; HCMV: Human Cytomegalovirus; HDs: Homology domains; HEK cells: Human embryonic kidney cells; HSV-1: Herpes simplex virus 1; ICP27: Infectious cell protein 27; ICTV: International Committee on Taxonomy of Viruses; IRS: Inverted repeated sequences; MHV-68: Murine Herpes Virus 68; NBCS: Newborn calf serum; NES: Nuclear export signal; NLS: Nuclear localization signal; NTRs: N-terminal regions; ORF: Open reading frame; PCR: Polymerase chain reaction; PPI: protein-protein interaction; UL: Unique long region; US: Unique short; Y2H: Yeast two-hybrid}

\section{Acknowledgements}

We would like to thank Ming Wen (College of Animal Science and Technology, Guizhou University) for kindly donating Y2H plasmids.

\section{Funding}

This work was supported by National Key Research and Development Program of China (2017YFD0500800), National Key R \& D Program (2016YFD0500800), China Agricultural Research System (CARS-42-17), and Sichuan Province Research Programs (2017JY0014/2017HH0026).

\section{Availability of data and materials}

Datasets used and/or analyzed in this study available from the corresponding author on reasonable request.

\section{Ethics approval}

The usage of duck embryo in this paper was approved by the Animal Ethics Committee of Sichuan Agricultural University (approval No. 2015-016).

\section{Authors' contributions}

$X G, R J$ and $A C$ conceived the study and designed the experiments; $X G$ performed the experiments; XG wrote the paper; RJ, MW, QY, SC, ML, YZ and AC guided the experiment and helped analysis of data. All authors read and approved the final manuscript.

\section{Consent for publication}

Not applicable.

\section{Competing interests}

The authors declare that they have no competing interests.

\section{Publisher's note}

Springer Nature remains neutral with regard to jurisdictional claims in published maps and institutional affiliations.

\section{Author details}

${ }^{1}$ Avian Disease Research Center, College of Veterinary Medicine, Sichuan Agricultural University, Chengdu, Sichuan 611130, China. ${ }^{2}$ Key Laboratory of Infectious Disease \& Bio-safety, Provincial Department of Education, Zunyi Medical University, Zunyi, Guizhou 563000, People's Republic of China. ${ }^{3}$ Institute of Preventive Veterinary Medicine, Sichuan Agricultural University, Chengdu, Sichuan 611130, China. ${ }^{4}$ Key Laboratory of Animal Disease and Human Health of Sichuan Province, Chengdu, Sichuan 611130, China.

Received: 23 March 2017 Accepted: 14 August 2017

Published online: 29 August 2017

\section{References}

1. Adams MJ, Lefkowitz EJ, King AMQ, et al. Ratification vote on taxonomic proposals to the International Committee on Taxonomy of Viruses (2016)[J]. Archives of virology. 2016;161(10):2921-49.

2. Fadly AM, Glisson JR, McDougald LR, Nolan L, DE S. Duck virus enteritis diseases of poultry. 12th ed; 2008. p. 384-93.

3. Wu Y, Cheng A, Wang M, Zhu D, Jia R, et al. Comparative genomic analysis of duck enteritis virus strains. J Virol. 2012;86:13841-2.

4. Wu Y, Cheng A, Wang M, Yang Q, Zhu D, et al. Complete genomic sequence of chinese virulent duck enteritis virus. J Virol. 2012;86:5965.

5. Wang J, Höper D, Beer M, Osterrieder N. Complete genome sequence of virulent duck enteritis virus (DEV) strain 2085 and comparison with genome sequences of virulent and attenuated DEV strains. Virus Res. 2011;160:316-25.

6. Li Y, Huang B, Ma X, Wu J, Li F, et al. Molecular characterization of the genome of duck enteritis virus. Virology. 2009;391:151-61.

7. He Q, Cheng A, Wang M, Xiang J, Zhu D, et al. Replication kinetics of duck enteritis virus UL16 gene in vitro. Virol J. 2012;9:1-4.

8. Xiang J, Ma G, Zhang S, Cheng A, Wang M, et al. Expression and intracellular localization of duck enteritis virus pUL38 protein. Virol J. 2010;7:162.

9. Chang H, Cheng A, Wang M, Zhu D, Jia R, et al. (2010) Research cloning, expression and characterization of $\mathrm{gE}$ protein of duck plague virus.

10. Lin M, Jia R, Wang M, Gao X, Zhu D, et al. Molecular characterization of duck enteritis virus CHv strain UL49. 5 protein and its colocalization with glycoprotein M. J Vet Sci. 2014;15:389-98.

11. Lin M, Jia R, Wang M, Gao X, Zhu D, et al. The transcription analysis of duck enteritis virus $U L 49.5$ gene using real-time quantitative reverse transcription PCR. Virus Genes. 2013;47:298-304.

12. Jacobson J, Martin S, Coen D. A conserved open reading frame that overlaps the herpes simplex virus thymidine kinase gene is important for viral growth in cell culture. J Virol. 1989;63:1839-43.

13. Nascimento R, Costa H, Dias J, Parkhouse R. MHV-68 open reading frame 20 is a nonessential gene delaying lung viral clearance. Arch Virol. 2011;156:375-86.

14. Kniżewski Ł, Kinch L, Grishin NV, Rychlewski L, Ginalski K. Human herpesvirus 1 UL24 gene encodes a potential PD-(D/E) XK endonuclease. J Virol. 2006;80:2575-7. 
15. Leiva-Torres GA, Rochette P-A, Pearson A. Differential importance of highly conserved residues in UL24 for herpes simplex virus 1 replication in vivo and reactivation. J Gen Virol. 2010;91:1109-16.

16. Blakeney S, Kowalski J, Tummolo D, DeStefano J, Cooper D, et al. Herpes simplex virus type 2 UL24 gene is a virulence determinant in murine and guinea pig disease models. J Virol. 2005;79:10498-506.

17. Jacobson JG, Chen S-H, Cook WJ, Kramer MF, Coen DM. Importance of the herpes simplex virus UL24 Gene for productive Ganglionic infection in mice. Virology. 1998;242:161-9.

18. Carvalho R, Spilki F, Cunha E, Stocco R, Arns C. Molecular data of UL24 homolog gene (ORF37) from Brazilian isolates of equine herpesvirus type 1. Res Vet Sci. 2012;93:494-7.

19. Kasem S, Yu MHH, Yamada S, Kodaira A, Matsumura T, et al. The ORF37 (UL24) is a neuropathogenicity determinant of equine herpesvirus 1 (EHV-1) in the mouse encephalitis model. Virology. 2010:400:259-70.

20. Lin S-R, Jiang MJ, Wang H-H, Hu C-H, Hsu M-S, et al. Human cytomegalovirus UL76 elicits novel Aggresome formation via interaction with S5a of the Ubiquitin Proteasome system. J Virol. 2013;87:11562-78.

21. Costa $H$, Nascimento $R$, Sinclair J, Parkhouse RME. Human cytomegalovirus gene UL76 induces IL-8 expression through activation of the DNA damage response. PLoS Pathog. 2013;9:e1003609.

22. Siew V-K, Duh C-Y, Wang S-K. Human cytomegalovirus UL76 induces chromosome aberrations. J Biomed Sci. 2009;16:107.

23. Nascimento R, Parkhouse R. Murine gammaherpesvirus 68 ORF20 induces cell-cycle arrest in $\mathrm{G} 2$ by inhibiting the Cdc2-cyclin B complex. J Gen Virol. 2007:88:1446-53.

24. Jackson SA, DeLuca NA. Relationship of herpes simplex virus genome configuration to productive and persistent infections. Proc Natl Acad Sci. 2003; 100:7871-6.

25. Lilley CE, Carson CT, Muotri AR, Gage FH, Weitzman MD. DNA repair proteins affect the lifecycle of herpes simplex virus 1. Proc Natl Acad Sci U S A. 2005;102:5844-9.

26. Strang BL, Stow ND. Circularization of the herpes simplex virus type 1 genome upon lytic infection. J Virol. 2005;79:12487-94.

27. Bertrand L, Leiva-Torres GA, Hyjazie H, Pearson A. Conserved residues in the UL24 protein of herpes simplex virus 1 are important for dispersal of the nucleolar protein nucleolin. J Virol. 2010;84:109-18.

28. Bertrand $\mathrm{L}$, Pearson $\mathrm{A}$. The conserved $\mathrm{N}$-terminal domain of herpes simplex virus 1 UL24 protein is sufficient to induce the spatial redistribution of nucleolin. J Gen Virol. 2008;89:1142-51.

29. Lymberopoulos $\mathrm{MH}$, Bourget A, Abdeljelil NB, Pearson A. Involvement of the UL24 protein in herpes simplex virus 1-induced dispersal of B23 and in nuclear egress. Virology. 2011;412:341-8.

30. Mongelard F, Bouvet P. Nucleolin: a multiFACeTed protein. Trends Cell Biol. 2007;17:80-6.

31. Rickards B, Flint S, Cole MD, LeRoy G. Nucleolin is required for RNA polymerase I transcription in vivo. Mol Cell Biol. 2007;27:937-48.

32. Jia R, Cheng A, Wang M, Zhu D, Ge H, et al. Cloning, expression, purification and characterization of UL24 partial protein of duck enteritis virus. Intervirology. 2009;52:326-34

33. Liu X, Liu Q, Xiao K, Li P, Liu Q, et al. Attenuated salmonella Typhimurium delivery of a novel DNA vaccine induces immune responses and provides protection against duck enteritis virus. Vet Microbiol. 2016;186:189-98.

34. Yu X, Jia R, Huang J, Shu B, Zhu D, et al. Attenuated salmonella typhimurium delivering DNA vaccine encoding duck enteritis virus UL24 induced systemic and mucosal immune responses and conferred good protection against challenge. Vet Res. 2012;43:1-10.

35. Malik P, Tabarraei A, Kehlenbach RH, Korfali N, Iwasawa R, et al. Herpes simplex virus ICP27 protein directly interacts with the nuclear pore complex through NUP62, inhibiting host nucleocytoplasmic transport pathways. J Biol Chem. 2012;287:12277-92.

36. Li M, Wang S, Xing J, Guo H, Zheng C. Molecular characterization of subcellular localization and nucleocytoplasmic shuttling of PRV UL54. BioMed Central Ltd. 2011;5:P78.

37. Guo H, Ding Q, Lin F, Pan W, Lin J, et al. Characterization of the nuclear and nucleolar localization signals of bovine herpesvirus-1 infected cell protein 27. Virus Res. 2009;145:312-20.

38. Tian X, Devi-Rao G, Golovanov AP, Sandri-Goldin RM. The interaction of the cellular export adaptor protein Aly/REF with ICP27 contributes to the efficiency of herpes simplex virus 1 mRNA export. J Virol. 2013;87:7210-7.

39. Fontaine-Rodriguez EC, Knipe DM. Herpes simplex virus ICP27 increases translation of a subset of viral late mRNAs. J Virol. 2008;82:3538-45.
40. Dai-Ju JQ, Li L, Johnson LA, Sandri-Goldin RM. ICP27 interacts with the Cterminal domain of RNA polymerase II and facilitates its recruitment to herpes simplex virus 1 transcription sites, where it undergoes proteasomal degradation during infection. J Virol. 2006;80:3567-81.

41. Gillis PA, Okagaki LH, Rice SA. Herpes simplex virus type 1 ICP27 induces p38 mitogen-activated protein kinase signaling and apoptosis in HeLa cells. J Virol. 2009:83:1767-77.

42. da Silva LF, Sinani D, Jones C. ICP27 protein encoded by bovine herpesvirus type 1 (blCP27) interferes with promoter activity of the bovine genes encoding beta interferon 1 (IFN- $\beta 1$ ) and IFN- $\beta 3$. Virus Res. 2012;169:162-8.

43. Bright H, Perez DL, Christy C, Cockle P, Eyles JE, et al. The efficacy of HSV-2 vaccines based on $\mathrm{gD}$ and $\mathrm{gB}$ is enhanced by the addition of ICP27. Vaccine. 2012;30:7529-35.

44. Jia R, Cheng A, Wang M, Qi X, Zhu D, et al. Development and evaluation of an antigen-capture ELISA for detection of the UL24 antigen of the duck enteritis virus, based on a polyclonal antibody against the UL24 expression protein. J Virol Methods. 2009;161:38-43.

45. Jia $R$, Cheng A, Wang $M$, Xin H, Guo Y, et al. Analysis of synonymous codon usage in the UL24 gene of duck enteritis virus. Virus Genes. 2009;38:96-103.

46. Mehla J, Caufield JH, Uetz P (2015) The yeast two-hybrid system: a tool for mapping protein-protein interactions. Cold Spring Harbor protocols 2015: pdb. top083345.

47. Mehla J, Caufield JH, Uetz P (2015) Mapping protein-protein interactions using yeast two-hybrid assays. Cold Spring Harbor protocols 2015: pdb. prot086157

48. Gao X, Jia R, Wang M, Zhu D, Chen S, et al. Construction and identification of a cDNA library for use in the yeast two-hybrid system from duck embryonic fibroblast cells post-infected with duck enteritis virus. Mol Biol Rep. 2014;41:467-75.

49. Phillips SL, Cygnar D, Thomas A, Bresnahan WA. Interaction between the human cytomegalovirus tegument proteins UL94 and UL99 is essential for virus replication. J Virol. 2012;86:9995-10005

50. Guan Y, Guo L, Yang E, Liao Y, Liu L, et al. HSV-1 nucleocapsid egress mediated by UL31 in association with UL34 is impeded by cellular transmembrane protein 140. Virology. 2014;464:1-10.

51. Corbin-Lickfett KA, Rojas S, Li L, Cocco MJ, Sandri-Goldin RM. ICP27 phosphorylation site mutants display altered functional interactions with cellular export factors Aly/REF and TAP/NXF1 but are able to bind herpes simplex virus 1 RNA. J Virol. 2010;84:2212-22.

52. Johnson LA, Sandri-Goldin RM. Efficient nuclear export of herpes simplex virus 1 transcripts requires both RNA binding by ICP27 and ICP27 interaction with TAP/NXF1, J Virol. 2009:83:1184-92.

53. Johnson LA, Li L, Sandri-Goldin RM. The cellular RNA export receptor TAP/ NXF1 is required for ICP27-mediated export of herpes simplex virus 1 RNA but the TREX complex adaptor protein Aly/REF appears to be dispensable. J Virol. 2009:83:6335-46.

54. Hardwicke MA, Sandri-Goldin RM. The herpes simplex virus regulatory protein ICP27 contributes to the decrease in cellular mRNA levels during infection. J Virol. 1994;68:4797-810

55. Ben Abdeljelil N, Rochette P-A, Pearson A. The UL24 protein of herpes simplex virus 1 affects the sub-cellular distribution of viral glycoproteins involved in fusion. Virology. 2013:444:263-73.

56. Fossum E, Friedel CC, Rajagopala SV, Titz B, Baiker A, et al. Evolutionarily conserved herpesviral protein interaction networks. PLoS Pathog. 2009;5: e1000570.

57. Liu C, Cheng A, Wang M, Chen S, Jia R, et al. Characterization of nucleocytoplasmic shuttling and intracellular localization signals in duck enteritis virus UL54. Biochimie. 2016;127:86-94. 